\section{A REVIEW OF INTER CELL INTERFERENCE MANAGEMENT IN REGULAR AND IRREGULAR NETWORKS GEOMETRY CELLULAR}

\author{
Rahat Ullah*, Fahim Ullah, Zubair Khalid, Hashim Safdar
}

Department of Electrical Engineering, Federal Urdu University of Arts, Science and Technology, Islamabad, 44000, Pakistan
Article history

Received

10 April 2021

Received in revised form

5 July 2021

Accepted

5 July 2021

Published online

20 August 2021

${ }^{*}$ Corresponding author dr.rahat@fuuastisb.edu.pk

\section{Graphical abstract}
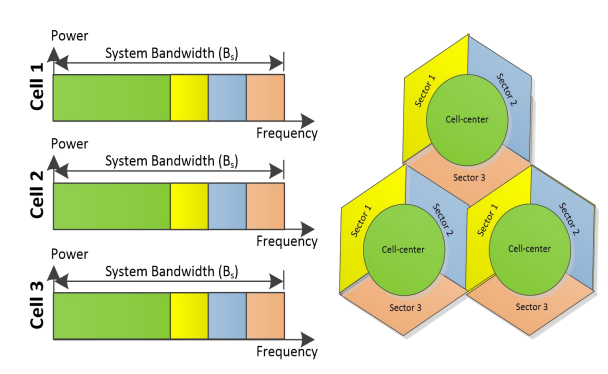

\begin{abstract}
This paper reviews the inter-cell interference (ICI) mitigation approaches in the OFDMA based multicellular networks with more emphasis on the frequency reused based $\mathrm{ICl}$ coordination schemes in the downlink systems. The geometry of the network severely affects the Signal to Interference and Noise Ratio (SINR); therefore, the wireless cellular systems are strongly dependent on the spatial BSs configuration and topology of a network. $\mathrm{ICI}$ mitigation techniques for both regular and irregular geometry networks are analyzed and a qualitative comparison along with the future research directions are presented.
\end{abstract}

Keywords: Inter-Cell Interference, OFDMA, Network Topology, Fractional Frequency Reuse, Irregular Geometry

(C) 2021 Penerbit UTM Press. All rights reserved

\subsection{INTRODUCTION}

Future cellular systems are rapidly advancing with the explosion of smart gadgets and broadband application services. In particular, data traffic demands have significantly increased in recent years, compelling the network operators to improve system capacity and coverage accordingly [1]. Consequently, effective radio resource management is acquiring significant consideration in the field of wireless communication [2]. This trend has prompted the development of new cellular technologies, of which fourth generation (4G) Long Term Evolution (LTE), Long Term Evolution - Advanced (LTE-A), and 5G are promising candidates. To accomplish high throughput and enhanced spectral efficiency these advanced standards are based on Orthogonal Frequency Division Multiple Access (OFDMA) [3] as a multiple access technique. With the integration of OFDMA, intracell interference is no more an issue due to orthogonal carriers for each user in the same cell, however, intercell interference
(ICI) is the primary source of interference and one of the prominent limiting factors in performance degradation in $4 G$ and $5 G$ systems [4], [5]. This problem of $\mathrm{ICl}$ is more severe at cell edges. To enhance the performance of the cellular network, Fractional Frequency Reuse (FFR) is an Inter-cell Interference Coordination (ICIC) approach, where each cell allocating its resources in a way such that to minimize the overall interference experienced in the network and to maximize the spatial reuse [6].

This paper reviews the performance of FFR as an interference mitigation scheme that has been investigated in literature for both regular geometry and irregular geometry models. In wireless cellular networks, the distance between the Base Station (BS) and the Mobile Station (MS) has a great impact on the SINR statistics. Therefore, the position of the mobile users concerning the co-channel BSs has a great effect on the interference experienced by that user. The SINR statistics of every single user corresponds to the location, its interference source positions along the sudden channel gain [7]. Accordingly, the geometry 
of the network has a significant effect on the SINR received. The performance of wireless cellular systems greatly depends on the spatial setup of BSs and also on the topology of a network [8]. Therefore, to analyze and design a cellular network, network geometry need to be considered [9].

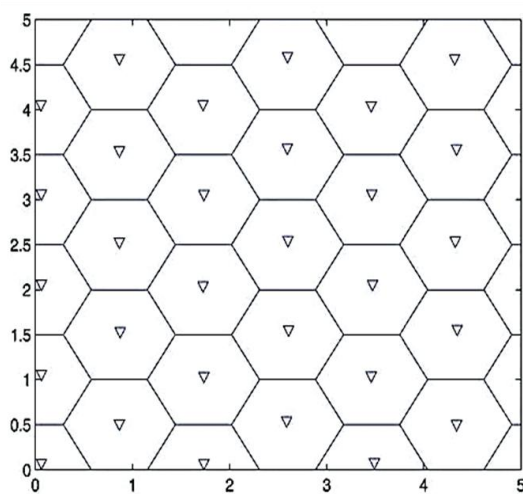

(a)

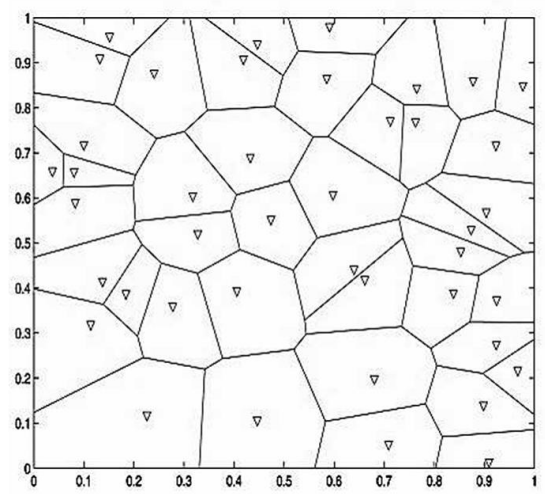

(b)

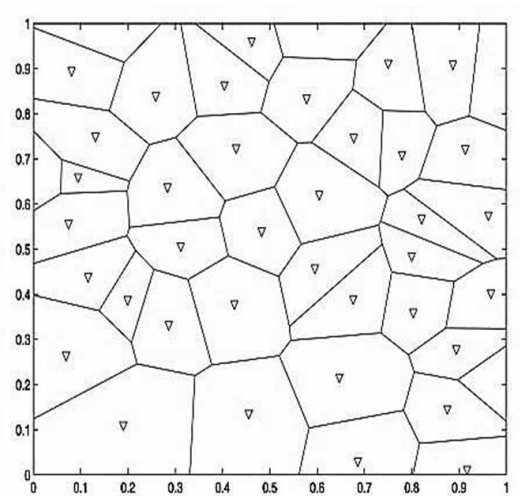

(c)

Figure 1 (a) Hexagon Grid Model (b) PPP Model (c) HCPP Model

Rest of the paper is organized as section 2.0 present a detailed description of the cellular network modeling and topologies. Section 3.0 gives the OFDMA implementation in 4G. ICl mitigation approaches in cellular networks are listed in section 14.0. A detailed description of the frequency reused-based $\mathrm{ICl}$ mitigation techniques is described in section 5.0. A review of the FFR schemes in the context of cellular geometry with detailed qualitative analysis is given in section 6.0. Section 7.0 concludes the future research directions.

\subsection{CELLULAR NETWORKS MODELING}

Generally, two types, regular and irregular geometrybased network modeling has been considered in the literature. In the regular geometry cellular network modeling, the BSs are assumed to be evenly positioned and follow a deterministic grid in the two-dimensional space. In perfect geometry models, equivalent quantities of neighboring cells are considered for every cell. For which the BSs are expected to be placed at ideal positions at the cell center. Examples of regular geometry models include the Hexagon Grid model and the Wyner model.

The hexagonal grid model as shown in Figure 1(a) is the generally acknowledged model and it has been broadly used in the literature for designing the typical cellular network. In hexagonal tessellation, each hexagon represents a cell in the network and each cell have the same coverage area. Moreover, mobile users are positioned either randomly or deterministically in the coverage area. The Wyner model is another extensively used model, to abstract the inter BSs interaction in the multi-cellular network. The Wyner model streamlines the inter-cell interference to one dimension and assumes an equal amount of interference from all the neighbor cells [10].
The generalization is acceptable when almost equal interference occurs due to the presence of a huge number of interference sources over the space [11]. When a cellular deployment is configured realistically, its layout is irregular, not just the channel situations change independently at each cell. Moreover, azimuths are also not aligned. Subsequently, every cell encounters different measures of $\mathrm{ICl}$ [12]. These facts have sparked the directions in research and presently models based on irregular cell geometry are picking up substantially higher consideration.

To meet the shortcomings of models based on regular cell geometry while considering realistic cellular deployment, Voronoi models based on irregular geometry have been anticipated for the tessellation. Voronoi models target the irregularity of the BS deployment along with the radio coverage for every BS. Moreover, in the stochastic geometry-based irregular geometry model, the position of the BS is modeled according to some Point Process (PP) [13]. A PP is a countable random collection of points. Recently, Poisson Point Process (PPP) [14] has been exploited in the literature to model cellular [15], cognitive, ad-hoc, and wireless sensor networks. The major limitation of PPP model is its self-contained assumptions [13], where two points (BSs) may be located closer to one another. Although, there are random distances among the BSs in cellular networks, yet it is impossible to find two BSs that closer to each other which are maintained by a similar operator. Consequently, the Mat'ern Hard Core Point Process (HCPP) being a hardcore factor that corresponds to the lowest adequate distance between two BSs can be considered in modeling the cellular network [16] as shown in Figure 1 (c). An HCPP can be defined as a repulsive point process with no two points of the process occurring within a parting distance lesser than a predefined hardcore parameter. Figure 1 exhibits the modeling of a wireless cellular network through the 
hexagonal grid (a), the PPP (b), and the HCPP (c), respectively.

\subsection{OFDMA IMPLEMENTATION IN $4 G$}

This section presents the technical aspects and fundamental features of OFDMA and its implementation in the $4 G$ cellular systems. To accomplish the requirement of increased throughput, coverage, and capacity, $4 \mathrm{G}$ cellular standards such as LTE [17] and LTE-A [18] have adopted OFDMA as their multiple access techniques for downlink only or both uplink and downlink communication. In wireless communication multipath propagation results in intersymbol interference (ISI), however, in the OFDMA system, it is no more an issuer because of the cyclic prefix [19]. Because of the OFDM modulation, the minimum sub-carrier spacing is required for an OFDMA radio transmission, which results in the maximum utilization of the spectrum band. In an OFDMA system, it is conceivable that adequately separated OFDM subcarriers can be allocated to the same user so that the channel is independent. This results in frequency diversity for a given user and provides robustness against the frequency selective channels. The radio channel condition of the sub-carriers is independent, that is sub-carriers fade independently for each user.

In OFDMA, it is possible to dynamically allocate the sub-carrier to the users based on their channel condition, this phenomenon is called multiuser diversity and it results in better utilization of the frequency spectrum in terms of capacity and throughput. OFDMA offers different transmission speeds for the different users simply by changing the number of allocated subcarriers to each user. Moreover, dynamic packet scheduling in OFDMA involves the process of decision making that which user is to transmit in which resources of the radio interface. The dynamic packet scheduling facility of the OFDMA efficiently explores the radio resource management in the OFDMA interface. Whereas it is also possible to efficiently exploit the multiuser diversity in the temporal domain. This is because in the multi-user scenario, the wireless channel undergoes independent fading and some of the users may experience good channel quality. Since the response of the channel is different for each user, therefore, it is possible to enhance the system efficiency in terms of capacity and throughput if the user transmission is properly scheduled.

In the 4G cellular systems, the BS is usually responsible for the packet scheduling which enables the scheduler to operate on a very short time scale (milliseconds). Inter-Cell Interference Coordination (ICIC) functionality of the LTE system offers coordination among the scheduler of the different cells to mitigate the inter-cell interference. Dynamiclink adaptation is the decision process of selecting the most appropriate modulation and coding scheme at any moment of the transmission. The general procedure of the OFDMA dynamic link adaptation is illustrated in Figure 2. Dynamic-link adaptation is based on the channel quality estimation at the BS through the channel quality information report by the user. The BS determines the modulation and coding scheme based on the channel estimation that ensures a certain Bit Error Rate (BER) depends upon the offered service and coding scheme. Generally, a link adaptation mechanism is implemented for the binary rate or throughput control by changing the users' allocated binary rate according to their channel quality estimation. Consequently, the users with good quality will observe a high data rate and vice versa.

The 3GPP LTE system utilizes OFDMA as a multiple access technology in the downlink radio interface. since in OFDMA the total bandwidth is divided into several sub-carriers, for LTE implementation the subcarrier spacing is $15 \mathrm{kHz}$. The sub-carriers are grouped into $180 \mathrm{kHz}$ blocks also called resource blocks which are comprised of 12 contiguous sub-carriers. LTE network deployment offers the flexibility in the choice from the widest variety of bandwidths of $\{1.4,3,5,10$, $15,20\} \mathrm{MHz}$, which corresponds to $\{6,15,25,50,75,100)$ resource blocks.

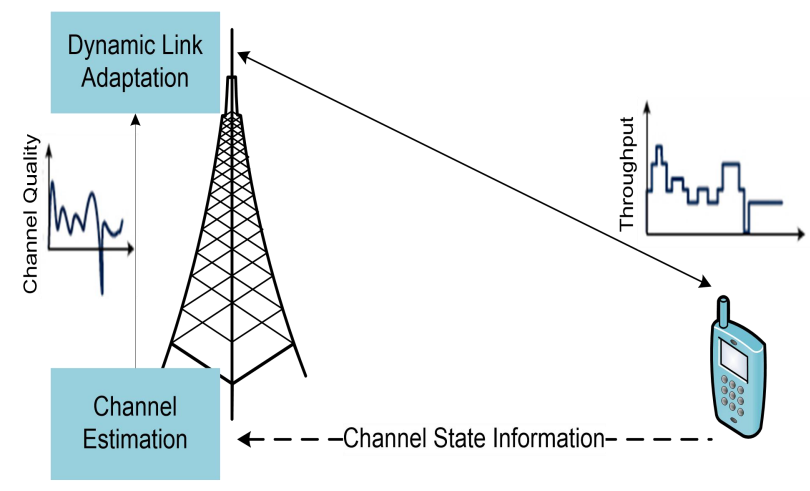

Figure 2 OFDMA Dynamic link adaptation

\subsection{ICI MITIGATION IN CELLULAR NETWORKS}

$\mathrm{ICl}$ has always been an issue with the cellular systems which evolves due to the dense reuse of radio resources and co-channel deployment. Therefore, the exploration of the $\mathrm{ICl}$ mitigation schemes has gained considerable attention to realize the dense spectrum reuse in the next-generation cellular standards [20]. Generally, the $\mathrm{ICl}$ mitigation schemes can be classed into three main groups, which are interference avoidance, interference cancellation, and interference randomization as shown in Figure 3. 


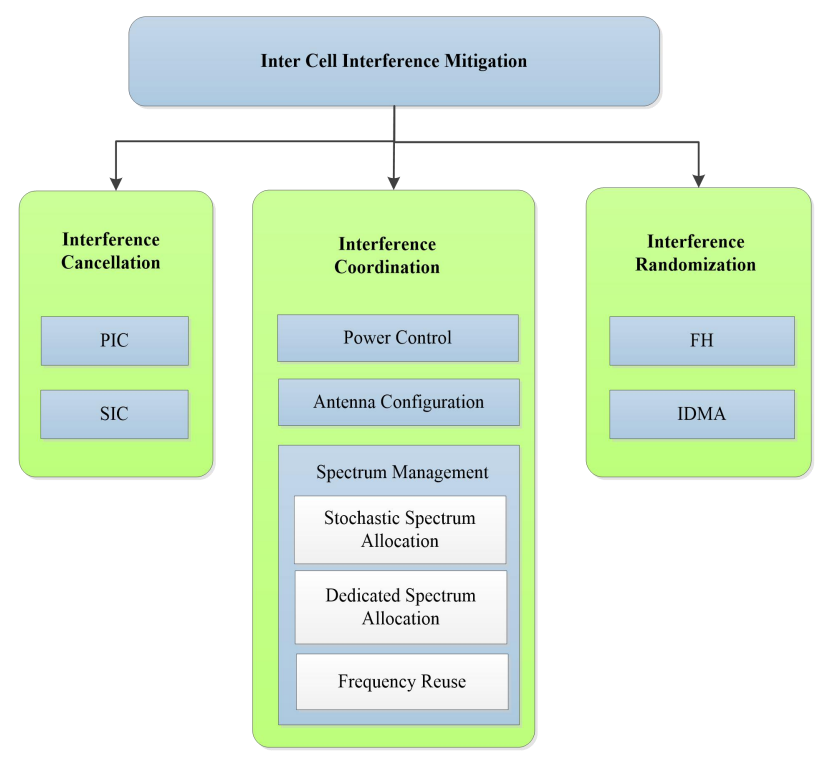

Figure $3 \mathrm{ICl}$ approaches in OFDMA systems

\section{A. Interference Cancellation}

An interference cancellation scheme is based on the principle of estimating and regenerating the interference signal at the receiver to be successively subtracted from the desired signal. The interference cancellation technique allows the interference to happen over the communication channel as it will be canceled at the receiver, therefore, this approach involves signal processing to reduce the interference at the receiver. The receivers are required to decode and demodulate the desired signal to extract useful information to be used with channel estimation for the interference cancellation.

The interference cancellation techniques available in the literature are grouped into two categories, filter-based approaches, and multi-user detection (MUD) based approaches. The first approach mitigates the interference using linear filers with the interference models, whereas the latter approach, MUD, involves signal processing in the decoding process. The interference cancellation promises considerable improvement in the system performance and can either be successive interference cancellation (SIC) [21] or parallel interference cancellation (PIC) [22]. The precise channel estimation capability of the interference cancellation has the potential to enhance the channel performance to that of the AWGN (Additive White Gaussian Noise) channel. Nevertheless, interference cancellation is usually applied in the uplink and considered less appropriate in the downlink because of its complexity in a process that rises exponentially according to the number of users. Moreover, this technique involves real-time information to be exchanged between the BSs to boost the system gain.

\section{B. Interference Randomization}

The interference randomization approach randomizes the interference also called the whitening or averaging by evenly distributing the interference among all users. The interference looks like the background noise with the interference randomization as it is averaged across the whole spectrum band. Frequency Hopping (FH) and the Interleave Division Multiple Access (IDMA) are examples of interference randomization.

The FH certifies that the user gets access to a certain range of channels to average the effect of interference for all the users. This approach is mostly applied in the CDMA-based cellular systems, however, in combination with interleaving, it is also induced to the FDMA/TDMA based systems. Moreover, FH and IDMA based interference randomization approaches are also proposed within the LTE standardization. Even though any additional signaling and measurement are not demanded in this technique, it causes some users to observe unnecessary interference even though if they were in good channel condition. Nonetheless, this approach randomizes the interference into AWGN, however, cannot significantly minimize the interference, and hence difficult to realize the substantial performance gain.

\section{Interference Coordination}

Interference coordination is correspondingly known as interference avoidance. Recently, interference coordination schemes have gained considerable attraction from both industry and academia, to combat the issue of $\mathrm{ICl}$. For mitigation of interference in the multicellular network, interference coordination is imposed constraints on the radio resource allocation through coordination among the neighbor cells. The radio resources to be utilized with coordination may be frequency, time, space, as well as transmit power. Interference coordination via constraint on the transmission power and space can be realized through power control [23] and antenna configuration, which includes beam-forming and Multiple Input and Multiple Output (MIMO) [24].

Nevertheless, interference coordination through power control is out of the scope here since this workemphasizes interference mitigation through spectrum management and uniform power distribution. However, the effect of antenna configuration is studied by changing the number of sector antennas at the cell edge region. $\mathrm{ICl}$ mitigation may be achieved through carefully considering the spectrum management approaches since the great cause of $\mathrm{ICl}$ is the spectrum collision. Spectrum management approaches for $\mathrm{ICl}$ mitigation include stochastic spectrum approaches [25], dedicated spectrum allocation approaches [26], and frequency reuse schemes [27]. 
In the literature, the stochastic spectrum and dedicated spectrum-based $\mathrm{ICl}$ coordination are mostly adopted in multi-tier networks. The stochastic spectrum allocation uses a probabilistic approach to allocate the frequency spectrum to a different tier of the network [28]. Whereas, the interference mitigation through the dedicated spectrum allocation method involves the spectrum partition in a way that nonoverlapping dedicated spectrum sub-bands are specified for particular cellular networks [29]. Frequency reuse-based $\mathrm{ICl}$ mitigation schemes in the literature, are discussed in detail in the next section. Figure 3 summarizes the above-mentioned $\mathrm{ICl}$ mitigation strategies.

The design framework for interference coordination via spectrum management approaches is generally grouped into two classes based on the degree of distribution. First, the centralized framework requires the central controller to execute a specific resource allocation and scheduling scheme to mitigate the $\mathrm{ICl}$. The interference mitigation technique in the central controller is executed following the information acquired from all the connected BSs. The information includes important parameters such as channel quality feedback reported by the user. The information is obtained through a signaling process at the central controller.

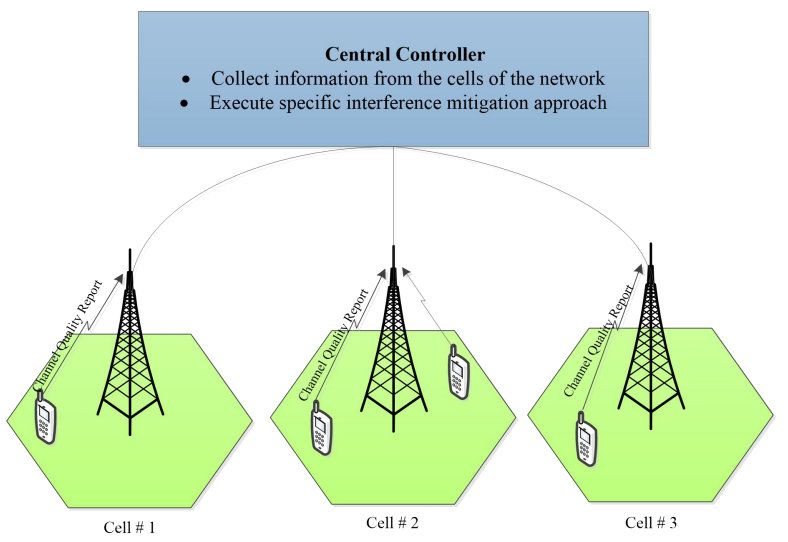

Figure 4 Centralized inter-cell interference mitigation framework
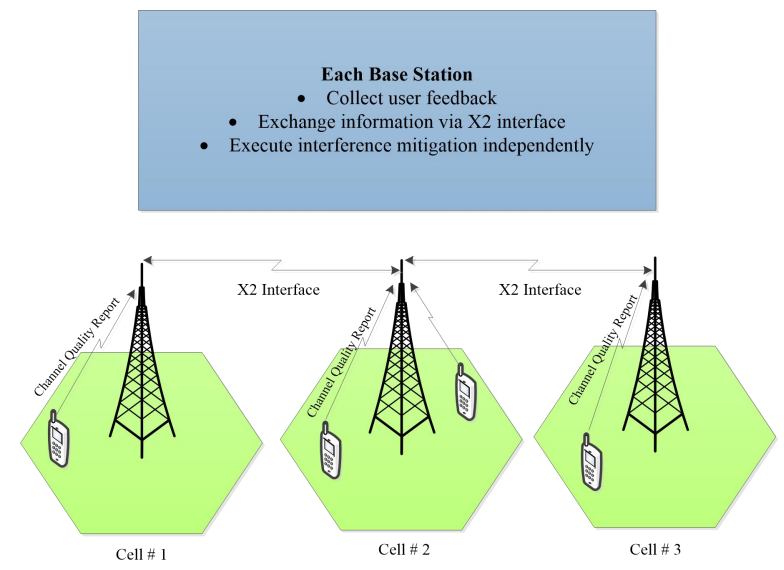

Figure $\mathbf{5}$ Decentralized inter-cell interference mitigation framework
The design framework of a centralized $\mathrm{ICl}$ mitigation scheme is presented in Figure 4. The exploitation of the network's global information allows the central controller to perform the $\mathrm{ICI}$ mitigation. The centralized interference mitigation approaches are more efficient with the lesser-dense cellular network. However, the dense deployment of the cellular network corresponds to the massive signaling between the BSs and the central controller [30]. Contrariwise, the decentralized approaches do not involve any centralized controller to execute the $\mathrm{ICl}$ mitigation scheme. The design framework of decentralized interference mitigation is elaborated in Figure 5. In this approach, the $\mathrm{ICl}$ mitigation is performed independently at every cell of the network. Therefore, less signaling overhead is experienced compared to the centralized approach. since the mechanism is based on inter-cell coordination, therefore the information is exchanged among the neighbor cell (via an X2 interface in the case of LTE). Moreover, the $\mathrm{ICl}$ coordination scheme can be cataloged into time scales, static, semi-static, and dynamic, as shown in Figure 6.

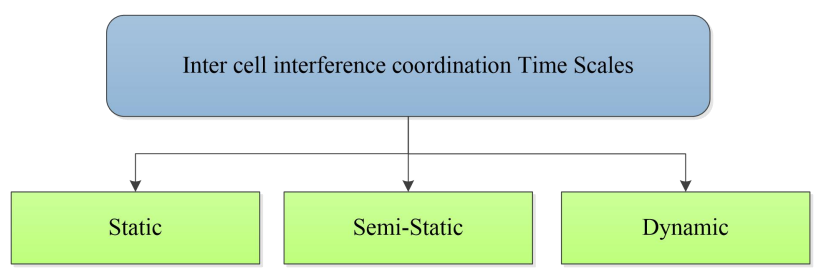

Figure 6 Categorize of ICl mitigation scheme

The static time scale-based inter-cell coordination schemes divide the total system spectrum resources into sub-resources or groups and allocates these resources for the total time [31]. The calculation of the resource allocation is executed once in the planning process. The part of the resources allocated to each cell, further sub-division of the allocated resources is fixed for different groups of users and will not be updated in the succeeding scheduling process. The long-term re-adjustments are executed through the network operation.

Semi-static-based $\mathrm{ICl}$ coordination schemes are quite similar to static approaches, however, in the semi-static the network parameter updates on demand. Part of the resources is permanently allocated while the remaining are allocated and updated according to the load condition at the cellcenter or cell-edge regions. Generally, the time required for the re-allocation in the semi-static approaches varies from a few hundred milliseconds to seconds. In the dynamic time scale interference coordination schemes, the system parameters are immediately adjusted whenever a change in the network condition is experienced. The resource allocation is adjusted following the traffic variations or load distribution [32]. Contrary to the static and semistatic approaches for interference avoidance, the 
resource allocation is repeatedly updated in a very short time scale.

\subsection{FREQUENCY REUSE BASED ICI MITIGATION SCHEMES}

To deal with the issue of inter-cell interference, one of the significant interference mitigation techniques is controlling the allocation of the spectrum resources over the multicellular network [33]. This can be realized through frequency reuse by applying a different frequency reuse factor (FRF) while allocating the system bandwidth.

The concept of FRF leads to frequency planning schemes that greatly reduce the inter-cell interference because the orthogonal spectrum sub-bands are distributed among the cells. The frequency reuse approaches as illustrated in Figure 5 and are generally classified as strict frequency reuse, fractional frequency reuse (FFR), and soft frequency reuse. Reuse- 1 and Reuse-3 are two different modes of strict frequency reuse. Similarly, FFR-3 also called sectoredFFR, is the modified form of the FFR scheme.

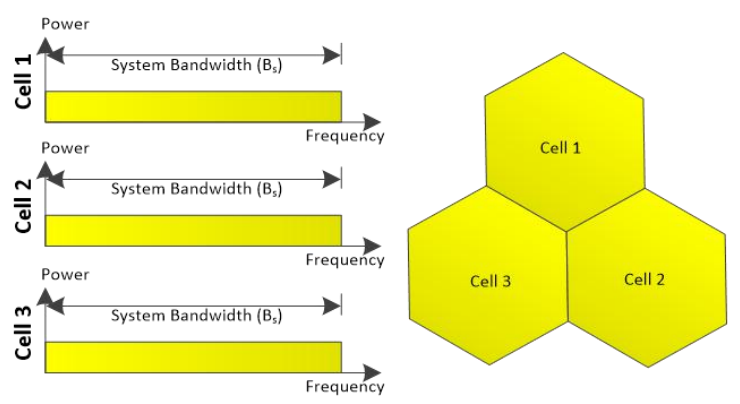

(a) Frequency Reuse-1 Scheme
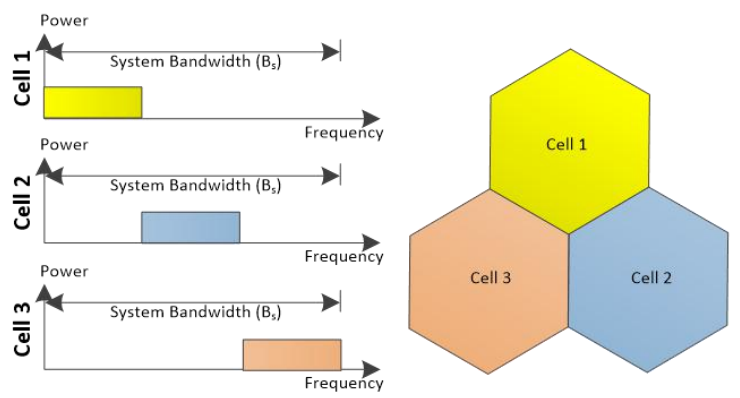

(b) Frequency Reuse-3 Scheme
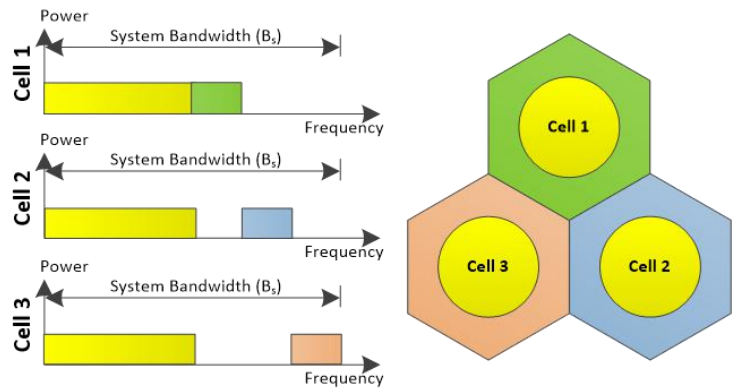

(c) Fractional Frequency Reuse Scheme
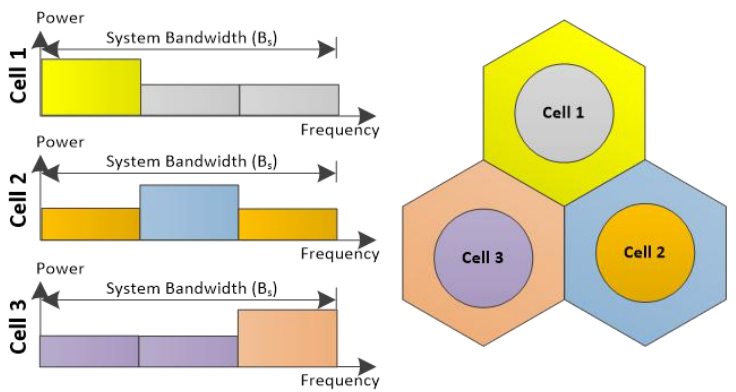

(d) Soft Frequency Reuse Scheme

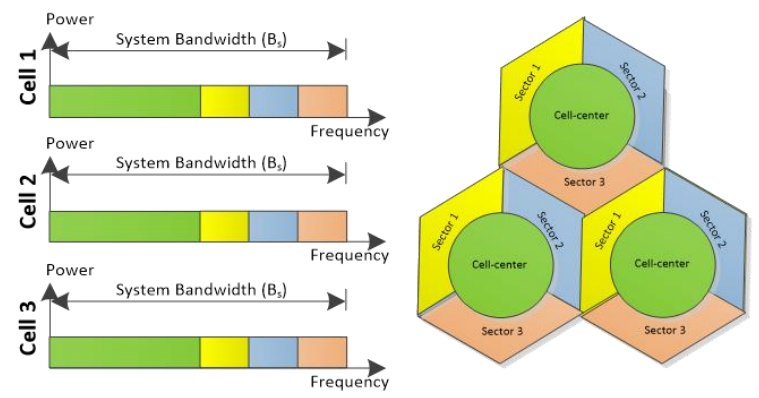

(e) Sectored-FFR Scheme

Figure 7 Frequency Reuse Schemes 


\section{A. Strict Frequency Reuse}

A strict frequency reuse scheme is also known as the Reuse-1 scheme is the simplest frequency allocation scheme shown in Figure 7(a), where the entire system bandwidth is accessible for any cell and can be reused at every cell of the multi-cellular network. Since all the cells is utilizing the same frequency spectrum, therefore each cell experiences severe interference. This $\mathrm{ICl}$ can be mitigated by increasing the FRF. The $F R F=M$, where $M>1$ indicates that the entire frequency spectrum is partitioned into $M$ sub-bands and allocated to $M$ cells of the network. FRF=3 corresponds to the Reuse-3 scheme shown in Figure $7(\mathrm{~b})$, which partitions the total spectrum into three equals size subbands to be strictly allocated to each cell of the cluster. The Reuse-3 scheme mitigates the $\mathrm{ICl}$ observed in Reuse-1, however, at the expense of low spectrum efficiency and hence the capacity [34]. This is because, in the Reuse-3, each cell of the network is assigned with only one-third of the total available spectrum.

\section{B. Fractional Frequency Reuse (FFR)}

From an influence point of view, all users within the same coverage area of a cell are not assumed to be similar. It occurs due to independent path loss effects and the channel situations for every user and also because of having different SINR values. As compared, the users at the cell edge are more sensitive to interference than those located in the cell center area. In FFR the cell area is divided into two sub-regions having the combined advantage of low and highfrequency users. Accordingly, the frequency spectrum is divided into two different parts. Part one being reused with the factor of one for every cell-center user. The other part is divided into many other sub-bands; where one of these sub-bands is assigned to the celledge region for each cell. FFR is a modified form of typical frequency reuse (FR) as shown in Figure 7 (c) for a hexagon model having cell-edge frequency reuse factor $M=3$. For each center area a shared frequency band is assigned while for cell-edge users, the bandwidth is cell-based and has a frequency reuse factor $\delta$. Therefore, in the FFR technique, there is a requirement of $M+1$ sub-bands. As the adjacent cells are assigned with the orthogonal sub-bands so none of a single spectrum is shared between the cell-center and cell-edge users. Therefore, the interference of celledge and cell-center users are significantly avoided.

\section{Soft Frequency Reuse (SFR)}

SFR scheme also requires the division of the cell coverage region into cell-center and cell-edge areas. In SFR, the total frequency spectrum is allocated to each cell of the network, although with a different power profile for the cell-center and cell-edge subbands. As illustrated in Figure $7(d)$, the cell-center region of one cell is assigned with an aggregate amount of frequency band that is allocated to the cell-edge region of the neighboring or adjacent cell. However, the cell-center region of the sub-band uses a low power level compared to the cell-edge region sub-band.

As for as spectrum efficiency is concerned, the SFR scheme is more bandwidth-efficient w.r.t the strict frequency reuse and FFR schemes, since SFR utilizes the entire system bandwidth at each cell of the network. However, the SFR scheme involves additional power control for the cell-center and cell-edge regions subbands [35].

\section{Sectored-FFR}

The FFR-3 scheme is the modified form of the FFR scheme [36], also known as the sectored-FFR scheme. The cell coverage region is divided into cell-center and cell-edge areas, where the cell-edge area is again partitioned into three more sectors. Sectoring of the cell-edge region diminish the number of the adjacent cell that can be the reason of interference in every sector as illustrated in Figure 7(e). The entire frequency spectrum is further partitioned into two more portions: part one is exclusively allocated to those users located in the cell-center region while the second part is divided into three more sub-bands and is assigned to the three sectors of the cell-edge area. The complexity implementation of the FFR based ICl schemes is much lower than that of SFR and therefore, it has been accepted as an attractive scheme for the multicellular systems based on OFDMA [37]. Approaches based on FFR in the multi-cellular deployment are reviewed in detail in the next section.

\subsection{REVIEW OF FFR APPROACHES}

As discussed, the induction of OFDMA as a radio access technique in cellular systems offers great spectral efficiency and flexibility in spectrum allocation [38]. However, in the next-generation cellular networks, the performance of the system is crucially hindered due to $\mathrm{ICl}$ because of the co-channel deployment or frequency reuse. The cellular system endeavors to avoid the collision between the co-channel deployed in the adjacent cells, by utilizing the $\mathrm{ICl}$ avoidance/coordination schemes. The interference coordination schemes in $4 G$ cellular systems characterize the frequency reuse procedures that restrict or allocate certain frequency resources among the users in the different cells. In the literature, different $\mathrm{ICl}$ coordination schemes are proposed with different traffic distribution and network structures. FFR is one of the techniques being accepted as an effective $\mathrm{ICl}$ coordination scheme in the $4 \mathrm{G}$ cellular systems such as LTE and LTE(Advanced) [39].

The idea of the FFR was proposed in [40] for the Global System for Mobile (GSM) communication network. Later this concept was adopted in the WiMAX [41] and 3GPP LTE cellular standards [42]. Recently, the 
FFR scheme is studied mostly in OFDMA based systems. Traditionally, the static bandwidth partitioning-based FFR scheme (also called strict-FFR) is adopted, where the total spectrum is partitioned into $\mathrm{N}+1$ sub-bands. Half of the total bandwidth of the system is assigned to the cell-enter region, whereas the remaining is divided into $N$ equal parts of size $(1 / N)$ and $N$ represents the number of adjacent cells in the region.

\section{A. FFR Approaches in Regular Geometry Cellular Networks}

In [6], a regular geometry-based FFR scheme with an enhanced scheduling technique is proposed to improve spectral efficiency. Cell partition is performed by considering a fixed distance as a threshold. Spectral efficiency has been improved; however, fullspectrum utilization is not achieved since no sectoring is considered at the cell edge region. The authors in [35] proposed that the spectrum assigned to the cellcenter region must be proportionate to the radius of the interior region. Subsequently, the leftover bandwidth is equally divided to be used in the celledge regions of the adjacent cells. The strict partitionbased approach is easy to implement, however, the frequency reuse configuration depends on preplanned spectrum partition and would remain fixed in the network deployment. Therefore, the FFR configuration with static spectrum partition would not be able to cope with the network variations because of the users' traffic distribution and their respective channel conditions. Consequently, the users with heavy traffic load regions endure insufficient spectrum resources, whereas the users from the lesser traffic demand region will be flooded with the excess of the spectrum resources.

The performance of FFR in terms of theoretical capacity and the outage rate for systems based on OFMA is studied in [43]. The joint effect of cell-partition and the spectrum allocation in the FFR scheme is formulated and optimized in the [44] for the OFDMA system. To overcome the limitations of static FFR configuration, dynamic spectrum partition-based FFR approaches are expected to the system spectrum efficiency. In [34], a dynamic spectrum partitionbased FFR (D-FFR) scheme is developed by Chang et. al. for the multicellular OFDMA network using a graph coloring approach. Their proposed scheme is centralized and assumed that the transmit power for the cell-edge region users be higher than that of the cell-center region users. In the response to the dynamic load conditions, an adaptive radio resource allocation is adopted in D-FFR by using a novel graph coloring approach. The proposed schemes are proven to modify the performance of the system in varying load conditions compared to the conventional strict FFR scheme. Similarly, in [38] optimal FFR scheme is proposed, where the sub-bands for the cell-center and cell-edge region users are selected based on user satisfaction. User satisfaction is the relative throughput that is calculated for a different combination of the cell-center region radius and frequency band allocation. After this calculation, the proposed scheme selects the optimal FFR deployment which multiplies the user satisfaction metric. Dynamic frequency planning-based FFR scheme for interference avoidance is proposed in [45]. The scheme works in a centralized manner and assumes a uniform distribution of the power transmission over the sub-bands acquired. The spectrum allocation problem in this scheme is disintegrated into two parts, to reduce the complexity of the FFR scheme in the multicellular network. First, the problem of the spectrum partition among the cellcenter and cell-edge regions is tackled via the central controller, then the problem of bandwidth assigned to the users of each region is solved independently by each BS in the network. Moreover, the minimum throughput required by each user is served by estimating the bandwidth demand of each user.

The authors in the [46] proposed a self-organized resource allocation (SORA) based FFR scheme which autonomously picks the optimal sub-bands for users at the cell-center and cell-edge regions. The optimization process is based on the coordination among the neighbor cells via a message exchange by the $\mathrm{x} 2$ interface over the LTE network. It is proved through the simulation that the suggested SORA scheme outperforms the traditional frequency reuse-1 and reuse-3 schemes in terms of cell-edge performance and fairness in resource allocation.

The major limitations of the traditional FFR schemes discussed so far in this section are the underutilization of the spectrum available entirely. Particularly, only $2 / 3^{\text {rd }}$ of the entire spectrum in each cell of the network, whereas the future cellular system aims to achieve frequency reuse-1 in each cell of the network to overcome the demands of constantly increasing data rate. To realize this aim of full-spectrum reuse at each of the networks, sectored-FFR also called the FFR-3 scheme has been adopted in the OFDMA based cellular system. 
Table 1 Summary of FFR Approaches

\begin{tabular}{|c|c|c|c|c|c|c|c|}
\hline Publication & $\begin{array}{l}\text { Cellular } \\
\text { Layout }\end{array}$ & Objective & $\begin{array}{c}\text { Centralized/ } \\
\text { Decentralized }\end{array}$ & $\begin{array}{l}\text { Spectrum } \\
\text { Allocation }\end{array}$ & $\begin{array}{c}\text { Cell- } \\
\text { Partition } \\
\text { Criteria }\end{array}$ & Sectoring & $\begin{array}{c}\text { FR-1 } \\
\text { Achieved }\end{array}$ \\
\hline [2] & Irregular (PPP) & Coverage & Decentralized & Dynamic & SINR & No & No \\
\hline [5] & $\begin{array}{l}\text { Regular } \\
\text { (Hexagon) }\end{array}$ & $\begin{array}{l}\text { Throughput } \\
\text { Maximization }\end{array}$ & Decentralized & Dynamic & Distance & No & No \\
\hline [27] & $\begin{array}{l}\text { Regular } \\
\text { (Hexagon) }\end{array}$ & $\begin{array}{l}\text { Throughput } \\
\text { Maximization }\end{array}$ & Decentralized & Dynamic & Distance & No & No \\
\hline [34] & $\begin{array}{l}\text { Regular } \\
\text { (Hexagon) }\end{array}$ & $\begin{array}{l}\text { Throughput } \\
\text { Maximization }\end{array}$ & Centralized & Dynamic & Distance & No & No \\
\hline [35] & $\begin{array}{l}\text { Regular } \\
\text { (Hexagon) }\end{array}$ & $\begin{array}{l}\text { Throughput } \\
\text { Maximization }\end{array}$ & Centralized & Static & Distance & No & No \\
\hline [36] & $\begin{array}{l}\text { Regular } \\
\text { (Hexagon) }\end{array}$ & $\begin{array}{l}\text { Throughput } \\
\text { Maximization }\end{array}$ & Decentralized & Dynamic & SINR & Yes & Yes \\
\hline [38] & $\begin{array}{l}\text { Regular } \\
\text { (Hexagon) }\end{array}$ & $\begin{array}{l}\text { User } \\
\text { Satisfaction }\end{array}$ & Centralized & Dynamic & Distance & No & No \\
\hline [45] & $\begin{array}{l}\text { Regular } \\
\text { (Hexagon) }\end{array}$ & $\begin{array}{l}\text { Throughput } \\
\text { Maximization }\end{array}$ & Centralized & Dynamic & Distance & Yes & Yes \\
\hline [46] & $\begin{array}{l}\text { Regular } \\
\text { (Hexagon) } 1\end{array}$ & $\begin{array}{l}\text { Throughput } \\
\text { Maximization }\end{array}$ & Decentralized & Dynamic & SINR & No & No \\
\hline [47] & $\begin{array}{l}\text { Regular } \\
\text { (Hexagon) }\end{array}$ & $\begin{array}{l}\text { Throughput } \\
\text { Maximization }\end{array}$ & Decentralized & Dynamic & Distance & No & No \\
\hline [48] & $\begin{array}{l}\text { Irregular } \\
\text { (perturbed) }\end{array}$ & Throughput & Centralized & Static & $\begin{array}{l}\text { Not } \\
\text { specified }\end{array}$ & No & No \\
\hline [49] & Irregular & $\begin{array}{l}\text { Throughput } \\
\text { maximization }\end{array}$ & Centralized & Dynamic & $\begin{array}{l}\text { Not } \\
\text { specified }\end{array}$ & No & Yes \\
\hline [50] & $\begin{array}{l}\text { Regular } \\
\text { (Hexagon) }\end{array}$ & $\begin{array}{l}\text { Throughput } \\
\text { Maximization }\end{array}$ & Decentralized & Dynamic & SINR & No & No \\
\hline [51] & Irregular (PPP) & Coverage & Centralized & Static & SINR & No & No \\
\hline [52] & Irregular (HPPP) & $\begin{array}{l}\text { Throughput } \\
\text { Maximization }\end{array}$ & Decentralized & Dynamic & SINR & Yes & Yes \\
\hline [53] & $\begin{array}{l}\text { Regular } \\
\text { (Hexagon) }\end{array}$ & $\begin{array}{l}\text { Throughput } \\
\text { Maximization }\end{array}$ & Centralized & Dynamic & Distance & Yes & Yes \\
\hline [54] & $\begin{array}{l}\text { Regular } \\
\text { (Hexagon) }\end{array}$ & $\begin{array}{l}\text { Throughput } \\
\text { Maximization }\end{array}$ & Decentralized & Dynamic & Distance & No & No \\
\hline
\end{tabular}

In [55] sectored-FFR scheme is proposed for OFDMA multicellular system to enhance the spectral efficiency. The coverage region of each cell is partitioned into the cell-center and cell-edge region and is further partitioned into three more sectors. The total spectrum is divided into two groups, a supergroup: to be used in the cell center region, and the regular group, which is again partitioned into three more subbands, which will be used in each of the three sectors at the cell-edge region. It is illustrated that the suggested sectored-FFR scheme enhances the data rate of the cell edge as well as the total cell throughput compared to the traditional FFR schemes. However, the static spectrum partition is considered in the proposed sectored-FFR scheme, the load distribution and user data rate demand have not been taken into consideration.

The performance of the sectored-FFR schemes is analyzed for the worst-case SINR in [56]. The proposed
sectored-FFR scheme is divided into two parts: the cellcenter and cell-edge region, where the cell-edge region is again divided into three more sectors by employing directional or sector antennas. The total system bandwidth divides into four equal portions, one for the cell-center region, while the remaining for the cell-edge region's three sectors. Moreover, users are proposed to be distributed uniformly in the cell. It is shown that the sectored-FFR scheme results in high spectral efficiency and a low probability of outage compared to the traditional FFR schemes. However, the heterogeneous user traffic demand is not considered equal sub-bands are allocated to every cell region, which is not in line with the practical cellular deployment.

In [47] optimal distance-based FFR scheme is analyzed for the downlink system with the aerial base station considering outage probability constraint and cell size. System-level design recommendations are 
concluded from the analytical results for initial FFR deployment in scalable cellular networks. A modified version of FFR is proposed in [57] with an extra intermediate region at the normal cell partition to avoid cross-tier interference. The proposed version of FFR is analyzed and it is shown that it outperforms the existing basic techniques in terms of cell throughput along with outage probability.

\section{B. FFR Approaches in Irregular Geometry Cellular Networks}

In the literature, most of the FFR approaches for interference avoidance assume that the location of the BSs follows the perfect geometry regular grid (hexagon grid model). In models with perfect geometry, a similar number of adjacent cells are targeted for every cell, in which the BSs are supposed to be located at an ideal position in the center of the cell. However, in the realistic deployment, the hexagon grid model is not followed by the BSs because of the disparity in the capacity demand across the network service area [13]. Consequently, the FFR scheme designed based on the unrealistic assumptions of the regular geometry models lead to inaccurate results [10]. These considerations have sparked the research directions and FFR schemes based on irregular cell geometry models have been proposed in the literature [58][59][60].

To investigate the limitations of the traditional FFR schemes the authors in [48] analyze the performance of the FFR scheme based on the irregular cellular layout. These BSs are considered to be perturbed from their ideal location, which is some degree of randomness is added to the ideal location of the BSs. Their result shows that the FFR $(1,4)$ scheme (where the cell-edge region frequency reuse factor is 4) gives better performance compared to $\operatorname{FFR}(1,3)$ or standard FFR. However, the proposed work has a major limitation in terms of spectrum utilization, that is only one-fourth of the cell-edge sub-band can be used at each cell. The authors in [49] proposed a Generalized Fractional Frequency Reuse (GFFR) based on flexible resource assignment to analyze the performance FFR scheme in the large-scale multicellular network with irregular cell geometry. The basis of the GFFR scheme is optimal sub-band and power allocation. The interference sensitivity is considered and adopted accordingly in the cell-edge spectrum and power allocation. The interference in the cell-edge regions with high interference sensitivity is minimized with subband isolation and power reduction. It is shown that the proposed GFFR scheme enhances system performance, particularly in the cell-edge region. However, there is no specific idea about the number of sub-band that would be assigned to every celledge region. Moreover, the required bandwidth by each user is also not considered.

In the irregular cell geometry models, the number of cells that can cause interference and the amount of co-channel interference are different for each cell of the network [59]. Therefore, the introduction of FFR into cell models based on irregular geometry is a complex issue. Due to random shapes of irregular models in Voronoi cells, it becomes difficult to set a criterion for the division of a cell into a cell center as well as the cell edge user. The standard distance-based cell partition in FFR cannot be directly applicable in the case of irregular geometry cells. Moreover, cell edges behave differently in w.r.t SINR levels and size[50]. However, the problem concerned with irregular cell partition is predicted in [58] for models based on PPP where users are classified into cell-center or cell-edge users based on an already set threshold SINR as compared to their actual space from the near serving BS. For both strict FFR and SFR having a frequency reuse factor of 3 located in the cell edge area, a flexible expression is derived for the coverage probability and coverage rate. The authors have also evaluated the tradeoffs among the two FFR schemes which are implemented on a model of irregular cell geometry. However, both [61] and [51] accounts for a simplistic case of the FFR, where both of the regions (cell-center and cell-edge) and also the sectoring of cells have not been considered [52]. In [62] it is shown that both FFR and SFR are potential interference management techniques in fifth-generation (5G) systems. A review of the literature is summarized in Table 1.

\subsection{CONCLUSION}

In this paper interference mitigation techniques based on frequency reuse for regular and irregular geometry, cellular networks are reviewed. FFR is an attractive $\mid \mathrm{Cl}$ mitigation scheme for multi-cell OFDMA networks such as LTE and LTE (Advanced). FFR is acknowledged because of its low complexity of implementation and its ability to efficiently utilize the scarce system spectrum. In literature, it is shown that the FFR scheme results in notable performance enhancement in terms of the network throughput, probability of outage, and cell-edge SINR. Therefore, it is needed to exploit the FFR scheme for $\mathrm{ICl}$ mitigation in the future multi-tier OFDMA network. In the literature, most of the FFR schemes are analyzed for perfect geometry hexagonal grid models. In which the BSs are supposed to be at the ideal location at each cell center and each adjacent cell is producing interference equally. Whereas, in wireless networks, the SINR experienced by each user depends on its location, positions of the interference sources, and instantaneous channel gains. The realistic deployment of the cellular network being irregular and has varying propagation conditions for each cell, therefore, a different quantity of $\mathrm{ICl}$ is experienced. Consequently, the SINR which the receiver experiences are highly dependent on the network geometry. These considerations inspire the inclusion of network topology based on irregular geometry in the designing of the FFR technique to mitigate interference in multicellular OFDMA systems. 
FFR has been studied as an ICI mitigation technique related to the cellular model based on irregular geometry. However, most of the schemes assume a static spectrum partition, which is not in line with the traffic demand of the users of the cell-center or celledge region. In the irregular geometry-based models, the cell partition (cell-center and cell-edge) leads to the sub-region with random coverage area because the cell structure is not regular. The cell partition in FFR results in sub-regions with a different number of users even the users are uniformly distributed. Consequently, each sub-region will have a different load condition, traffic demands, and spectrum requirement. Therefore, instead of static spectrum partition-based sub-band allocation in the FFR configuration, a dynamic spectrum partition-based FFR scheme will be an excellent fit. The dynamic sub-band partition will be able to cope with the load or traffic variation in cellular deployment. In literature, the irregularity in the BSs deployment is captured by using the homogeneous PPP. The major limitation of PPP related to FFR is its independent assumption, in which the two BSs may be possibly closer to each other. While there is a random distance among the BSs when the cellular network is deployed realistically. Therefore Mat'ern Hard Core Point Process (HCPP) being a repulsive PP having hardcore parameters reflecting the suitable and least distance between the two BSs is the better choice in modeling the topology of the cellular network. Furthermore, with previous research, where FFR into a model based on irregular geometry is concerned only with simplistic FFR (strict FFR) with cell-center and celledge regions while sectoring of cell is not considered. In the strict-FFR approaches only a single part of the band of cell edge is assigned to users in that section, therefore results in lower spectral utilization. Whereas 5G cellular systems are targeting full frequency reuse (Reuse-1) in all the cells of the network along with heterogeneous multi-tier deployment. Therefore, to fully utilize the available spectrum in each cell of the network, the sectored-FFR scheme along with the optimal resource allocation for the individual users of heterogeneous multi-tier networks is a potential technique to mitigate interference in the future $5 \mathrm{G}$ cellular networks.

\section{References}

[1] F. Ali, H. Yigang, G. Shi, Y. Sui, and H. Yuang. 2020. Future Generation Spectrum Standardization for $5 \mathrm{G}$ and Internet of Things. J. Commun. 15(3): 276-282.

[2] S. C. Lam and K. Sandrasegaran. 2020. Fractional Frequency Reuse in Multi-tier Networks: Performance Analysis and Optimization. Int. J. Wirel. Inf. Networks. 27(1): 164-183.

[3] A. Idris, S. S. Md Yusoff, N. Fadzlina Naim, S. S. Sarnin, M. Kassim, and M. D. Rozaini. 2019. Reduction of Inter-Cell Interference (ICI) by Fractional Frequency Reuse (FFR) in Orthogonal Frequency Division Multiple Access (OFDMA). 2019 International Conference on Information and Communications Technology (ICOIACT). 123-128.

[4] G. Nardini et al. 2018. Practical Feasibility, Scalability and
Effectiveness of Coordinated Scheduling Algorithms in Cellular Networks Towards 5G. J. Netw. Comput. Appl. 106: $1-16$.

[5] B. J. Veancy and P. Yogesh. 2020. Fractional Frequency Reuse with Enhanced Scheduling Strategies. Wirel. Pers. Commun. 0123456789.

[6] B. J. Veancy and P. Yogesh. 2021. Fractional Frequency Reuse with Enhanced Scheduling Strategies. Wirel. Pers. Commun. 117(3): 2541-2553.

[7] M. Anannya and R. M. Shourov. 2018. Performance Measurement Model of Mobile User Connectivity in Femtocell/Macrocell Networks using Fractional Frequency Re-use Scheme. Int. J. Adv. Comput. Sci. Appl. 9(5): 355-362.

[8] J. J. G. Andrews, R. K. R. Ganti, S. W. M Haenggi, N Jindal, and N. J. S. W. M Haenggi. 2010. A Primer on Spatial Modeling and Analysis in Wireless Networks. IEEE Commun. Magzine. November: 156-163.

[9] R. Ullah, N. Fisal, H. Safdar, Z. Khalid, and W. Maqbool. 2015. Fractional Frequency Reuse for Irregular Geometry Based Heterogeneous Cellular Networks. 5th Natl. Symp. Inf. Technol. Towar. New Smart World. 5-10.

[10] J. XU, J. Zhang, and J. G. Andrews. 2011. On the Accuracy of the Wyner Model in Downlink Cellular Networks. IEEE Int. Conf. Commun. 1-5.

[11] A. J. Viterbi, A. M. Viterbi, and E. Zehavi. 1994. Other-cell Interference in Cellular Power-controlled CDMA. IEEE Trans. Commun. 42(2-4): 1501-1504.

[12] D. G. González et al. 2011.On the Performance of Static Inter-cell Interference Coordination in Realistic Cellular Layouts. Mob. Networks Manag. Springer. 02: 163-176.

[13] H. ElSawy, E. Hossain, and M. Haenggi. 2013. Stochastic Geometry for Modeling, Analysis, and Design of Multi-Tier and Cognitive Cellular Wireless Networks: A Survey. IEEE Commun. Surv. Tutorials. 15(3): 996-1019.

[14] F. Baccelli and B. Blaszczyszyn. 2012. Stochastic Geometry and Wireless Networks Applications. INRIA \&Ecole Norm. Super. Paris. II.

[15] C.-H. Lee, C.-Y. Shih, and Y.-S. Chen. 2012. Stochastic Geometry Based Models for Modeling Cellular Networks in Urban Areas. Wirel. Networks. 19(6): 1063-1072, Oct.

[16] A. Guo and M. Haenggi. 2013. Spatial Stochastic Models and Metrics for the Structure of Base Stations in Cellular Networks. IEEE Trans. Wirel. Commun. 1-12.

[17] S. Stefania, T. Issam, and B. Matthew. 2009. LTE, The Umts Long Term Evolution: From Theory to Practice. 6.

[18] J. Wannstrom. 2012. LTE-Advanced. 3GPP. May.

[19] M. Einhaus and O. Klein. 2006. Performance Evaluation of a Basic OFDMA Scheduling Algorithm for Packet Data Transmissions. Proc. Int. Symp. Comput. Commun. 695-701.

[20] A. A. Hafez, Y. M. Jaamour, and K. I. Khorzom. 2019. Resource Allocation in OFDMA Femtocell Based LTE and 5G Networks with QoS Guarantees. J. Eng. Appl. Sci. 15(2): 643652.

[21] J. Axnäs, Y. P. E. Wang, M. Kamuf, and N. Andgart. 2011. Successive Interference Cancellation Techniques for LTE Downlink. IEEE Int. Symp. Pers. Indoor Mob. Radio Commun. PIMRC. 1793-1797.

[22] K. Sakoda, H. Hata, and S. Hata. 2020. Residue Effect of Parallel Interference Canceller in Belief Propagation Decoding in Massive MIMO Systems. Int. J. Electr. Electron. Eng. Telecommun. 9(1): 13-17.

[23] S. H. Kang and J. G. Kim. 2020. Spectrum Allocation with Power Controlin LBS based D2D Cellular Mobile Networks. J. Commun. 15(3): 283-288.

[24] D. Pankong, V. Imtawil, and P. Suthisopapan. 2019. BER Performance of Cooperative MIMO Systems with Halfduplex Decode and Forward Relaying. Int. J. Electr. Electron. Eng. Telecommun. 8(5): 268-273.

[25] G. A. Zhang, J. Y. GU, Z. H. Bao, C. XU, and S. B. Zhang. 2013. Interference Management for LTE-Advanced Het-Nets: Stochastic Scheduling Approach in Frequency Domain. Trans. Emerg. Telecommun. Technol. 25(3): 294-307.

[26] W. Jeon, J. Kim, and D. Jeong. 2014. Downlink Radio Resource Partitioning with Fractional Frequency Reuse in 
Femtocell Networks. IEEE Trans. Veh. Technol. 63(1): 308-321.

[27] A. H. Alquhali, M. Roslee, M. B. Abdelgadir, K. Kordi, and K. S. Mohamed. 2020. Enhanced Fractional Frequency Reuse Approach for Interference Mitigation in Femtocell Networks. Bull. Electr. Eng. Informatics. 9.

[28] S. Liu, Y. Chang, G. Wang, and D. Yang. 2012. Distributed Resource Allocation in Two-hierarchy Networks. ETRI J. 34(2): 159-167.

[29] C. Kyongkuk, L. Wooju, Y. Dongweon, H. Kwangmin, and Y. S. Choi. 2009. Resource Alloation for Orthogonal and Cochannel Femtocells in a Hierarchical Cell Structure. IEEE Int. Conf. Consum. Electron. 655-656.

[30] F. B. Álvarez. 2010. Contribution to Dynamic Spectrum Assignment in Multicell OFDMA Networks A Self-Organization and Machine Learning Approach. April: 181.

[31] D. González G., M. García-Lozano, S. Ruiz, and J. Olmos 2010. Static Inter-cell Interference Coordination Techniques for LTE Networks: A Fair Performance Assessment. 3th Int. Conf. Mult. Access Commun. 211-222.

[32] S. Elayoubi. 2008. Performance Evaluation of Frequency Planning Schemes in OFDMA-based Networks. IEEE Trans. 7(5): 1623-1633.

[33] A. S. Hamza, S. S. Khalifa, H. S. Hamza, and K. Elsayed. 2013. A Survey on Inter-cell Interference Coordination Techniques in Ofdma-based Cellular Networks. IEEE Commun. Surv. Tutorials. 15(4): 1642-1670.

[34] R. Y. Chang, Z. Tao, J. Zhang, and C. C. J. Kuo. 2013. Dynamic Fractional Frequency Reuse (D-FFR) for Multicell OFDMA Networks Using a Graph Framework. Wirel. Commun. Mob. Comput. 13(1): 12-27.

[35] T. Novlan, J. G. Andrews, I. Sohn, R. K. Ganti, and A. Ghosh. 2010. Comparison of Fractional Frequency Reuse Approaches in the OFDMA Cellular Downlink," IEEE Glob. Telecommun. Conf. GLOBECOM 2010. 1-5.

[36] J. He, W. Guan, W. Guo, W. Liu, and W. Cheng. 2019. Analytical Evaluation of Cellular Network Uplink Communications with Higher Order Sectorization Deployments. IEEE Trans. Veh. Technol. 68(12): 12179-12189.

[37] N. Saquib, E. Hossain, and D. Kim. 2013. Fractional Frequency Reuse for Interference Management in LTEadvanced Hetnets. IEEE Wirel. Commun. 20(2): 113-122.

[38] D. Bilios, C. Bouras, V. Kokkinos, A. Papazois, and G. Tseliou. 2013. Selecting the Optimal Fractional Frequency Reuse Scheme in Long Term Evolution Networks. Wirel. Pers. Commun. 71 (4): 2693-2712.

[39] S. A. Khan, A. Kavak, s. Aldirmaz Çolak, and K. Küçük. 2019. A Novel Fractional Frequency Reuse Scheme for Interference Management in LTE-A HetNets. IEEE Access. 7: 109662-109672.

[40] K. Begain, G. Istvan Rozsa, A. Pfening, and M. Telek. 2002. Performance Analysis of GSM Networks with Intelligent Underlay-overlay. Proc. - IEEE Symp. Comput. Commun. 135-141.

[41] WiMAX Forum. 2006. Mobile WiMAX Part I: A Technical Overview and Performance Evaluation. WiMAX Forum; Technical Report.

[42] 3GPP TSG RAN WG1\#42 R1-050764, Inter-cell interference handling for E-UTRA, Ericsson, Technical Report. 2008.

[43] H. Fujii and H. Yoshino. 2010. Capacity and Outage Rate of OFDMA Cellular System with Fractional Frequency Reuse. IEICE Trans. Commun. E93-B(3): 670-678.

[44] M. Assaad. 2008. Optimal Fractional Frequency Reuse (FFR) in Multicellular OFDMA System. 2008 IEEE 68th Veh. Technol. Conf. 1-5.

[45] S. H. Ali and V. C. M. Leung. 2009. Dynamic Frequency Allocation in Fractional Frequency reused OFDMA Networks. IEEE Trans. Wirel. Commun. 8(8): 4286-4295.
[46] S. Mohamed, M. Abd-Elnaby, and S. A. El-Dolil. 2015. Selforganized Dynamic Resource Allocation Using Fraction Frequency Reuse Scheme in Long Term Evolution Networks. Computers \& Electrical Engineering. 48: 174-186. Doi:10.1016/j.compeleceng.2015.04.018. https://www.sciencedirect.com/science/article/abs/pii/S00 45790615001469.

[47] S. Chang, S. Kim, and J. P. Choi. 2020. The Optimal Distance Threshold for Fractional Frequency Reuse in Size-Scalable Networks. IEEE Trans. Aerosp. Electron. Syst. 56(1): 527-546.

[48] P. Mitran and C. Rosenberg. 2012. On Fractional Frequency Reuse in Imperfect Cellular Grids. IEEE Wireless Communications and Networking Conference, WCNC. 2967-2972.

[49] L. Chen and D. Yuan. 2012. Generalizing and Optimizing Fractional Frequency Reuse in Broadband Cellular Radio Access Networks. EURASIP J. Wirel. Commun. Netw. 2012(1): 230.

[50] S. R. Boddu, A. Mukhopadhyay, B. V. Philip, S. S. Das, and R. V. Rajakumar. 2014. Bandwidth Partitioning and SINR Threshold Analysis of Fractional Frequency Reuse in OFDMA Cellular Networks for Real Time and Best Effort Traffic. Wirel. Pers. Commun. 75: 325-347.

[51] T. D. Novlan et al. 2011. Analytical Evaluation of Fractional Frequency Reuse for OFDMA Cellular Networks. IEEE Trans. Wirel. Commun. 10(12): 4294-4305.

[52] R. Ullah, H. Ullah, Z. Khalid, and H. Safdar. 2020. Irregular Geometry Based Sectored FFR Scheme for $\mathrm{ICl}$ Mitigation in Multicellular Networks. J. Commun. 15(11): 796-807.

[53] M. Amer. 2012. Optimal Configuration of Fractional Frequency Reuse System for LTE Cellular Networks. Veh. Technol. Conf. (VTC Fall), 2012. 1.

[54] G. Sahu and S. S. Pawar. 2020. An Approach to Reduce Interference Using FFR in Heterogeneous Network. SN Comput. Sci. 1(2): 1-6.

[55] W. Theories. 2007. A Novel Multi-cell OFDMA System Structure Using Fractional Frequency Reuse. IEEE International Symposium on Personal, Indoor and Mobile Radio Communications (PIMRC'07) A. 1-5.

[56] S. Hashima, H. Shalaby, M. Alghoniemy, O. Muta, and H. Furukawa. 2013. Performance Analysis of Fractional Frequency Reuse based on Worst Case Signal to Interference Ratio in OFDMA Downlink Systems. IEEE 24th Int. Symp. Pers. Indoor, Mob. Radio Commun. 616-620,

[57] K. Sakoda et al. 2019. Efficient Resource Allocation with Improved Interference Mitigation in FFR-aided OFDMA Heterogeneous Networks. J. Electron. Sci. Technol. 17(1): 7389.

[58] T. T. D. Novlan et al. 2012. Analytical Evaluation of Fractional Frequency Reuse for Heterogeneous Cellular Networks. IEEE Trans. Commun. 60(7): 2029-2039,

[59] R. Ullah et al. 2014. Stochastic Geometry Based Dynamic Fractional Frequency Reuse for OFDMA Systems. J. Teknol. 67(1): 61-67.

[60] R. Ullah, N. Fisal, H. Safdar, W. Maqbool, Z. Khalid, and A. S. Khan. 2013. Voronoi Cell Geometry Based Dynamic Fractional Frequency Reuse for OFDMA Cellular Networks. IEEE Int. Conf. Signal Image Process. Appl. 435-440.

[61] T. D. Novlan, R. K. Ganti, J. G. Andrews, and A. Ghosh. 2011. A New Model for Coverage with Fractional Frequency Reuse in OFDMA Cellular Networks. IEEE Glob. Telecommun. Conf. - GLOBECOM 2011. 1-5.

[62] E. M. Soultan, H. B. Nafea, and F. W. Zaki. 2020. Interference Management for Different 5G Cellular Network Constructions. Wirel. Pers. Commun. 0123456789. 